\title{
Representación en el Núcleo de la Esencia de Semat de las Competencias de un Equipo de Desarrollo de Software
}

\author{
Claudia E. Durango-Vanegas ${ }^{(1,3)}{ }^{*}$, Carla M. Zapata-Rueda ${ }^{(2)}$ y Carlos M. Zapata-Jaramillo ${ }^{(3)}$ \\ (1) Facultad de Ingeniería, Universidad de San Buenaventura, Cra. 56C \#51-110, Medellín-Colombia \\ (e-mail: claudia.durango@usbmed.edu.co; cedurangov@unal.edu.co) \\ (2) Facultad de Psicología, Universidad de San Buenaventura, Cra. 56C \#51-110, Medellín-Colombia \\ (e-mail: carla.zapata@usbmed.edu.co) \\ (3) Facultad de Minas, Departamento de Ciencias de la Computación y de la Decisión, Universidad Nacional de Colombia, \\ Av. 80 \#65 - 223, Medellín-Colombia (e-mail: cmzapata@unal.edu.co)
}

Recibido Oct. 12, 2018; Aceptado Dic. 18, 2018; Versión final Feb. 14, 2019, Publicado Ago. 2019

\begin{abstract}
Resumen
En este artículo se propone la representación, en el núcleo de la Esencia de Semat, de las competencias de los principales roles de los equipos de desarrollo de software. En diversas propuestas se describen los perfiles de los equipos de software, pero falta definir las competencias y niveles de competencia según el rol de las personas en el ciclo de desarrollo de software. Para lograrlo se integra la teoría de competencias organizacionales con el núcleo de la Esencia de Semat, definiendo roles y competencias de personas con habilidades para trabajar en equipos de desarrollo de software. Como resultado se obtienen siete competencias con niveles de competencia, adicionales a las existentes en el núcleo de la Esencia de Semat para cinco roles principales de los equipos de desarrollo de software. Se espera que la representación propuesta ayude a las empresas desarrolladoras de software a conformar equipos de trabajo eficientes y con rendimientos excepcionales.
\end{abstract}

Palabras clave: competencia organizacional; desarrollo de software; destreza; equipo de trabajo; Semat

\section{Representation of the Competencies of a Software Development Team based on the Semat Essence Kernel}

\begin{abstract}
In this paper we propose a representation of the competencies of the main roles of the software development teams in the Semat Essence kernel. Some proposals include the profiles of the software teams, but a definition of competencies and levels of competency according to the role of the people in the software development life cycle is still needed. To achieve this, we integrate the theory of organizational competencies with the Semat Essence kernel, for identifying roles and competencies of people with skills of teamwork in software development. As a result, seven competencies with their level of competencies represented in the Semat Essence kernel, additional to the existing representations of the roles involved in the software development life cycle, are obtained. It is expected that software development companies will consider the proposed competencies and the levels of competencies in order to construct efficient software development teams with exceptional performance.
\end{abstract}

Keywords: organizational competency; software development; ability; work team; Semat 


\section{INTRODUCCIÓN}

Las competencias son conocimientos, habilidades y destrezas que desarrolla una persona para comprender, transformar y participar en el mundo en el que vive, por medio de la capacidad efectiva para llevar a cabo exitosamente una actividad plenamente identificada (Furnham, 2001). Esta capacidad se define y mide en términos del desempeño en un determinado contexto. Las competencias son elementos dinámicos que se pueden generar, potenciar, apoyar y promover (por ser inseparables de la acción a ejecutar) y exigen conocimiento (Cristancho et al., 2006). El núcleo de la Esencia de Semat (Teoría y Método de la Ingeniería de Software, según siglas en inglés) es una iniciativa que permite realizar la representación gráfica y textual de las prácticas comunes de los métodos de desarrollo de software, mediante el uso de un grupo de elementos del núcleo. Estos elementos (entre ellos las competencias) son esenciales y universales para todo esfuerzo de desarrollo de software. Por ello, en Semat se definen las competencias como las habilidades clave requeridas y presentes en los miembros del equipo de trabajo para desarrollar software (Jacobson et al., 2013).

Las personas que conforman equipos de desarrollo de software tienen habilidades específicas porque tienden a comportarse de manera regular y distintiva según el logro de las actividades y debido a la forma en que colaboran e influencian el desempeño del equipo de trabajo (Estrada y Peña, 2013; Quezada y Mengual, 2018). En consecuencia, varios autores (López y André, 2006; Da Silva, 2013; Matturro, 2013; Estrada et al., 2013; Rodríguez et al., 2013; Barrera, Barrera y Hernández, 2015; Matturro, 2015; Bano et al., 2016; Keshav, Pawar y Ramrakhiyani, 2016; Mtsweni et al., 2016) describen los roles de las personas que integran equipos de desarrollo de software, buscando valorar las habilidades específicas de las personas. En estas propuestas se busca conformar equipos de trabajo con competencias diferenciadoras para optimizar los métodos de trabajo que respondan a las demandas del medio, sin tener en cuenta las competencias y los niveles de competencia de las personas según el rol que desempeñan en los equipos de desarrollo de software.

Por ello, en este artículo se propone la integración de la teoría de competencias de la psicología organizacional (que busca establecer equipos de alto rendimiento y con disposición para afrontar nuevos desafíos) con los aportes existentes en el núcleo de la Esencia de Semat relacionados con las competencias de un equipo de desarrollo de software. Por ello, se proponen siete competencias adicionales y los niveles de competencia encontrados en un grupo de personas que conforman el equipo de desarrollo de software, caracterizándolas y representándolas con los elementos del núcleo de la Esencia de Semat. La representación se basa en los principales roles de un equipo de desarrollo de software, tales como: director del proyecto, coordinador del proyecto, analista, desarrollador y tester. Como resultado, se espera que la representación en el núcleo de la Esencia de Semat de las competencias y los niveles de competencia propuestos se usen en empresas desarrolladoras de software para conformar equipos de trabajo eficientes y con rendimientos excepcionales. Además, se espera que sea posible medir la capacidad productiva y el desempeño de las personas según el rol que ocupan en los equipos de desarrollo de software.

Las competencias son características subyacentes de las personas y se relacionan con el comportamiento y desempeño para resolver situaciones y desafíos laborales. Una competencia organizacional se considera la unión de aptitudes, actitudes, rasgos de personalidad y conocimientos requeridos para lograr un estándar de efectividad y de desempeño superior en un trabajo o situación específica. Por ello, Spencer y Spencer (1993) definen el modelo de competencias denominado "Modelo del Iceberg". En el modelo se plantea que las personas tienen dos tipos de competencias: genéricas o visibles (fáciles de identificar) y específicas o no visibles (difíciles de identificar). Las competencias genéricas son conocimientos y destrezas que las personas adquieren mediante capacitaciones para desarrollar una tarea concreta. Las competencias específicas son características individuales relacionadas con comportamientos observables y habituales que posibilitan el éxito de las personas y con rasgos de personalidad y motivaciones para ejecutar una tarea concreta, teniendo en cuenta el conocimiento personal (Spencer y Spencer, 1993; Durango et al., 2015). Según lo anterior, las competencias organizacionales son capacidades de las personas para lograr un buen desempeño en contextos complejos y auténticos, basándose en la integración y activación de conocimientos, habilidades, destrezas, actitudes y valores.

Jacobson et al. (2014) definen las competencias para participar en un esfuerzo de ingeniería de software como contenedores genéricos de habilidades específicas. Además, estos autores mencionan que una persona dentro de un equipo de trabajo requiere una competencia relevante para las tareas específicas y otras competencias para la comprensión. A continuación, se presentan las seis competencias existentes en el núcleo de la Esencia de Semat (véase la Figura 1; Jacobson et al., 2014): representación del interesado (habilidad para recopilar y comunicar necesidades del interesado, con un conocimiento completo del dominio del cliente); análisis (habilidad para descubrir oportunidades, necesidades y solicitudes del interesado, convirtiéndolas en los requisitos acordados para el sistema de software); desarrollo (habilidad para implementar sistemas basados en requisitos, utilizando programación); pruebas (habilidad para verificar que 
el software desarrollado atiende los requisitos y la calidad suficiente, utilizando pruebas de software); liderazgo (habilidad para conducir un equipo hacia una conclusión exitosa, que satisfaga a los interesados); gestión (habilidad para coordinar, planear y rastrear el trabajo que realiza el equipo).

Las competencias en el núcleo de la Esencia de Semat se agrupan en tres áreas de interés (véase la Figura 1; Jacobson et al., 2014): cliente que contiene todo lo que se relaciona con el uso actual y la explotación del sistema de software que se produce; solución que contiene todo aquello que se relaciona con las especificaciones y el desarrollo del sistema de software; esfuerzo que contiene todo aquello que se relaciona con el equipo de desarrollo y la manera en que hacen el trabajo.

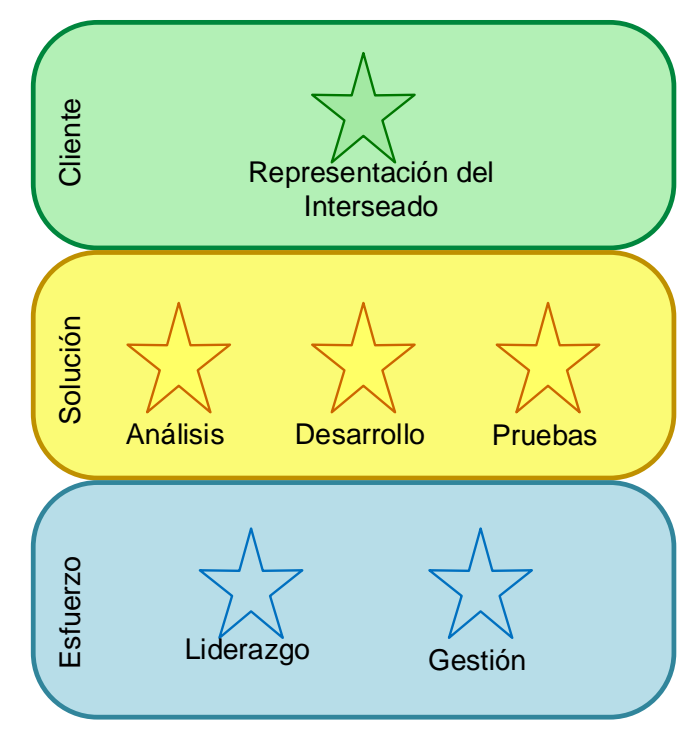

Fig. 1: Competencias del núcleo de la Esencia de Semat. (Traducido de Jacobson et al., 2013; OMG, 2015)

\section{ANTECEDENTES}

Rodríguez et al. (2013) proponen un perfil ideal en equipos de trabajo en proyectos de desarrollo de software, identificando tres elementos básicos para su conformación: tamaño, personalidad y competencias genéricas. Los autores consideran que los proyectos de desarrollo de software tienen grupos pequeños de personas dedicados a actividades específicas. Esta conformación busca optimizar el potencial de las personas según la experiencia en resolver situaciones similares y la complejidad de las actividades. Por lo anterior, los autores proponen tamaños de los equipos de trabajo fluctuantes entre tres y siete personas y detectan problemas en la integración de equipos de trabajo debido a las diversas características de personalidad. Además, proponen cuatro roles principales de los miembros de equipos de desarrollo de software: implementador, creativo, coordinador y monitor evaluador. La selección del rol adecuado depende de las cualidades de personalidad como creatividad, disciplina, cumplimiento de logros, adaptación y liderazgo. En relación con las competencias, los autores distinguen dos tipos de competencias: genéricas y específicas. Las competencias genéricas se refieren a las características de comportamiento general de las personas, independientemente de los conocimientos tecnológicos; las competencias específicas se asocian con conocimientos y habilidades tecnológicas del rol (Rodríguez et al., 2013). Los autores definen un perfil ideal en equipos de desarrollo de software según el tamaño y los patrones de comportamiento y definen algunas competencias genéricas y específicas de los roles identificados. Sin embargo, les hace falta identificar y asociar las competencias para cada uno de los roles identificados y definir los niveles de competencia.

Da Silva et al. (2013) proponen unos criterios de selección de equipos de trabajo para proyectos de desarrollo de software. Los autores realizan una serie de entrevistas a 14 empresas de desarrollo de software de Brasil, analizando un total de 88 proyectos de software. Entre los criterios resultantes se encuentran: la composición y efectividad de los equipos de trabajo, la percepción exitosa en proyectos agiles, conocimiento y habilidades del equipo de trabajo, nivel de madurez de la empresa y niveles de replicación de los métodos de desarrollo aplicados en los proyectos (Da Silva et al., 2013). Los criterios que proponen los autores se relacionan con aspectos de gestión de proyectos, aunque les falta incorporar las competencias de cada uno de los roles que conforman un equipo de desarrollo de software.

Matturro et al. (2013) presentan una propuesta de habilidades blandas presentes en equipos de desarrollo de software. Las habilidades blandas son capacidades interpersonales que demuestran la destreza de las personas para comunicarse y construir relaciones con otras personas. Estas habilidades blandas permiten 
mejorar la comunicación, la negociación, la resolución de problemas, la resolución de conflictos y la toma de decisiones, entre otros. Para identificar las habilidades blandas, los autores recolectan datos de encuestas en 11 empresas de desarrollo de software en Uruguay, presentando una lista de 17 habilidades blandas (Matturro, 2013) de 678 artículos científicos. En trabajos posteriores, listan las habilidades blandas que se deben considerar para los roles de analista de negocios, director de proyecto, desarrollador, tester y usuario final. Además, concluyen que hay una amplia variedad de habilidades blandas en un equipo de desarrollo de software que dependen del rol y que son vitales para el desempeño de sus actividades (Matturro, 2013; Matturro et al., 2015; Mtsweni et al., 2016). Los autores presentan un listado de habilidades blandas para los roles importantes en el desarrollo de software. Sin embargo, les falta definir y considerar los niveles aceptables de cada habilidad blanda.

Durango et al. (2015) proponen una representación en el núcleo de la Esencia de Semat de las competencias de ingeniería social necesarias para mejorar la seguridad informática. Ingeniería social es una ciencia relacionada con la interacción social para persuadir a individuos u organizaciones con el propósito de obtener información privada. Para obtener las competencias del atacante de ingeniería social, los autores aplican pruebas psicotécnicas 16PF-5 a un grupo de personas con habilidades de ingeniería social para identificar sus rasgos de personalidad. Como resultado, se obtiene un listado de competencias genéricas y específicas con los descriptores de las competencias de ingeniería social (véase la Tabla 1). Posteriormente, representan estas competencias en el núcleo de la Esencia de Semat considerando las áreas de interés de Semat (véase la Figura 2; Durango et al., 2015; Durango, Amariles y Zapata, 2017). Los autores consideran que las competencias genéricas y específicas de una persona con habilidades de ingeniería social pueden apoyar a los tester a mejorar la seguridad informática. Además, representan las competencias identificadas en la Esencia de Semat. Sin embargo, falta identificar las competencias y niveles de competencia de los principales roles de un equipo de desarrollo de software, tales como desarrollador, analista, director y coordinador.

Tabla 1: Competencias genéricas y específicas con sus descriptores del Tester de software, para prevenir ataques informáticos que utilizan ingeniería social. (Adaptado de Durango, Amariles y Zapata, 2017)

\begin{tabular}{|l|l|l|}
\hline $\begin{array}{c}\text { Tipo de } \\
\text { competencia }\end{array}$ & \multicolumn{1}{|c|}{ Nombre } & \multicolumn{1}{c|}{ Descriptores } \\
\hline \multirow{5}{*}{ Genérica } & Dominio tecnológico & $\begin{array}{l}\text { Manejo de redes, entrenamiento técnico y tecnológico, } \\
\text { conocimiento tecnológico, dominio de software y búsquedas en } \\
\text { redes }\end{array}$ \\
\cline { 2 - 3 } & Trabajo corporativo & Formación de equipos y relación interpersonal \\
\cline { 2 - 3 } & Negociación & Dominio del negocio, adaptabilidad y liderazgo \\
\cline { 2 - 3 } & Comunicación estratégica & Comunicación efectiva y asertiva, oratoria y redacción \\
\cline { 2 - 3 } & Pensamiento sistémico & $\begin{array}{l}\text { Adaptabilidad, innovación, retrospección y procesamiento } \\
\text { paralelo }\end{array}$ \\
\cline { 2 - 3 } & Flexibilidad & Proactividad, adaptabilidad, apertura y creatividad \\
\hline \multirow{5}{*}{ Específica } & Solución de problemas & Iniciativa, proactividad y toma de decisiones \\
\cline { 2 - 3 } & Seguimiento y control & $\begin{array}{l}\text { Acompañamiento, profundización, validación, evaluación y } \\
\text { mejoramiento }\end{array}$ \\
\cline { 2 - 3 } & Capacidad de influenciar & Persuasión, convencimiento, empatía, credibilidad y confianza \\
\cline { 2 - 3 } & Capacidad de respuesta & Agilidad, oposición, exigencia y procesamiento paralelo \\
\hline
\end{tabular}
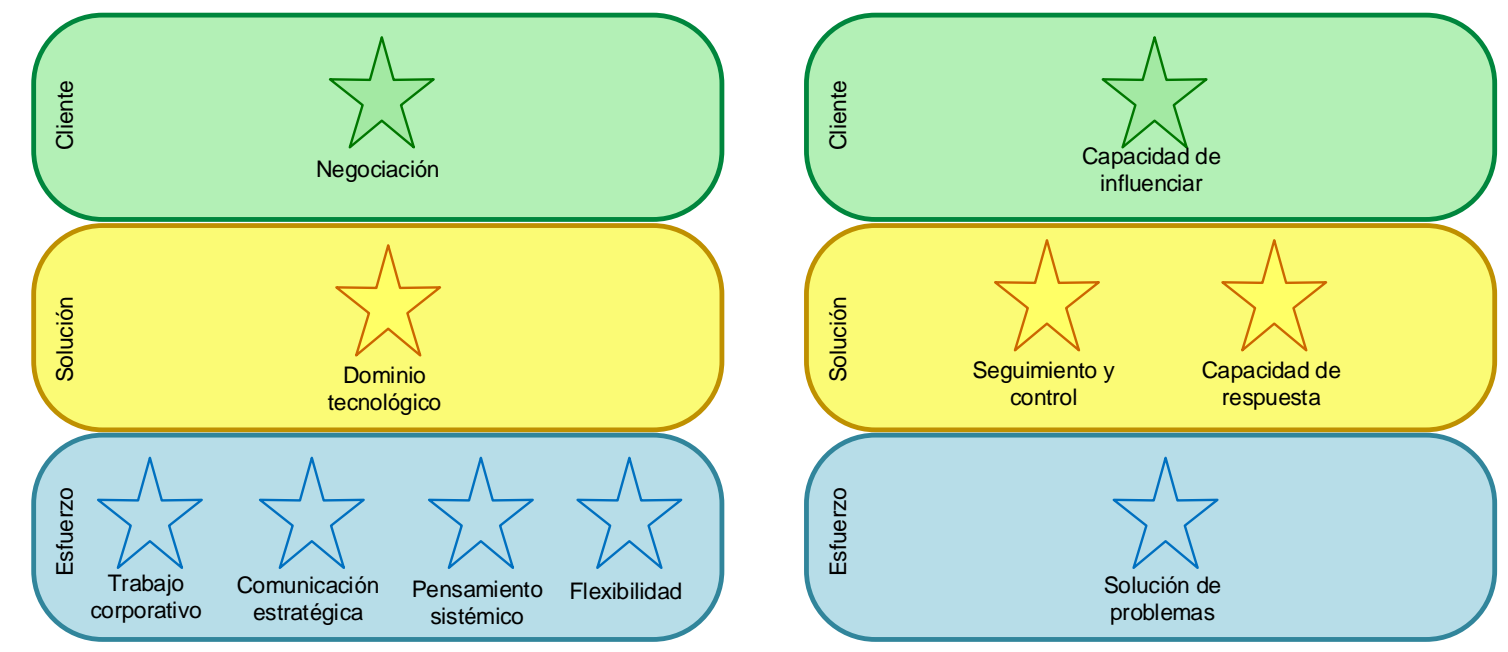

Fig. 2: Representación de las competencias genéricas y específicas del atacante de ingeniería social en la Esencia de Semat. (Tomado de Durango, Amariles y Zapata, 2017) 


\section{COMPETENCIAS ORGANIZACIONALES}

En esta Sección se propone una integración de la teoría de competencias organizaciones de la psicología organizacional (que busca establecer equipos de alto rendimiento y con disposición para afrontar nuevos desafíos) con los aportes del núcleo de la Esencia de Semat relacionados con las competencias de un equipo de desarrollo de software. Para la ejecución del proyecto se cuenta con tres grupos de actores: ingenieros, actores externos y psicólogos. El Ingeniero es la persona con el conocimiento de los métodos de desarrollo de software (fases, actividades y productos de trabajo, entre otros), de los roles del equipo de desarrollo de software y de las competencias para representar en el núcleo de la Esencia de Semat las competencias organizacionales. Los actores externos son los profesionales que ocupan los roles identificados para el proyecto de investigación. El psicólogo es la persona con el conocimiento para identificar las competencias organizacionales y ejecutar las pruebas psicológicas (Licorish y MacDonell, 2015). En la Figura 3 se presenta un diagrama de actividades del proyecto:

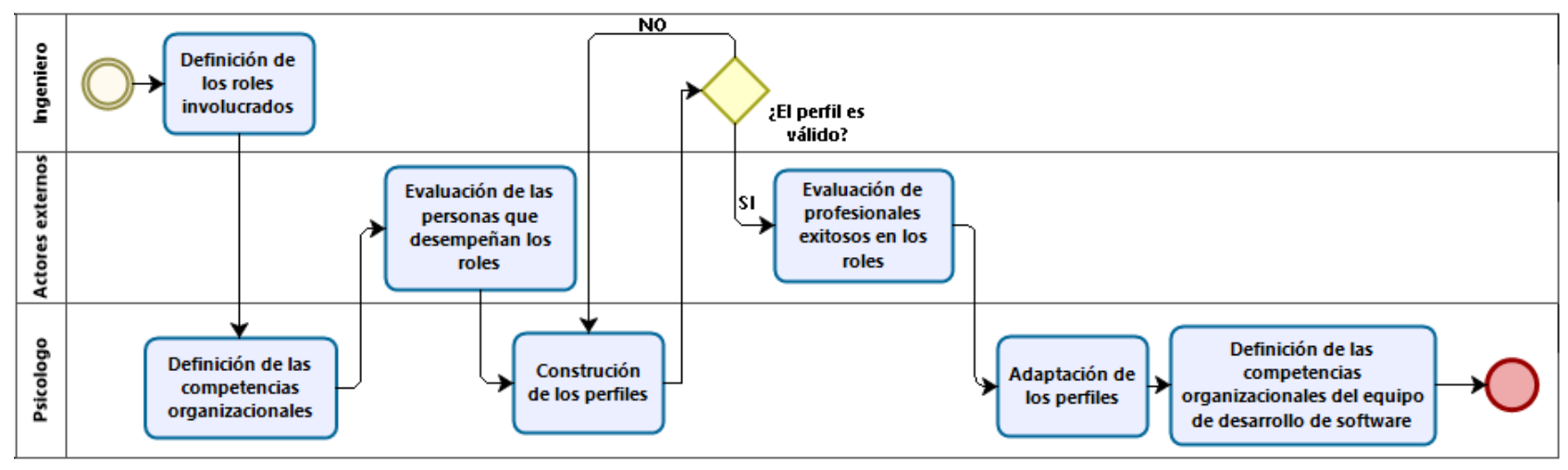

Fig. 3: Diagrama de actividades del proyecto "Competencias Semat de un equipo de desarrollo de software"

\section{Definición de los roles involucrados}

Los roles se definen mediante las responsabilidades a cumplir y un conjunto de actividades relacionadas que se asocian con un tipo de trabajo, donde una persona puede ocupar uno o varios roles o un rol puede contar con varias personas. La asignación de un rol en un equipo de trabajo depende del propósito del proyecto, las tareas a ejecutar y los productos requeridos (Rodríguez et al., 2013; Keshav, Pawar y Ramrakhiyani, 2016). A continuación, se presentan las definiciones de los cinco principales roles en un equipo de desarrollo de software:

Director de Proyecto: es el responsable de definir los objetivos y metas del proyecto, garantizando su continuidad y gestionando los recursos necesarios para la culminación del proyecto. El director del proyecto establece las condiciones de trabajo, dirige y aprueba las actividades del proyecto (Rodríguez et al., 2013).

Coordinador del Proyecto: es el responsable de atender las necesidades de los miembros del equipo de trabajo, brindando soluciones a los requisitos que soliciten. El coordinador de proyecto asume la responsabilidad de los éxitos y fracasos del equipo de desarrollo y la aceptación del producto de parte del interesado. Además, lidera la planeación del proyecto, aplicando los conocimientos y las técnicas que permitan al equipo alcanzar los resultados deseados (Gallego y Hernández, 2015).

Analista: es el responsable de diseñar el sistema y está en constante contacto con los miembros del equipo de trabajo, revisando el correcto desarrollo de las especificaciones del proyecto. El analista identifica las necesidades del interesado, convirtiéndose en un consultor del negocio y proponiendo mejoras y soluciones. El analista es el vínculo entre el interesado y el equipo de desarrollo, representando los intereses del cliente y los resultados de los miembros del equipo (López y André, 2006).

Desarrollador: es el responsable de definir y mantener el código fuente de los componentes del sistema de software, garantizando la funcionalidad requerida en el proyecto. El desarrollador verifica y evalúa el grado de cumplimiento y coherencia de la aplicación según las directrices y lineamientos del proyecto. El desarrollador está en constante comunicación con el director y coordinador del proyecto para resolver inconvenientes y revisar los resultados que surgen durante la ejecución del proyecto (López y André, 2006).

Tester: es el responsable de verificar y comprobar la calidad de los resultados que propone el equipo de desarrollo de software. El tester identifica, define e implementa las pruebas necesarias para contrastar y analizar los resultados obtenidos en el proyecto, garantizando el cumplimiento de los requisitos del usuario y del producto final (López y André, 2006). 


\section{Definición de las competencias organizacionales}

La identificación de las competencias organizacionales resulta de un proceso investigativo en el que se utiliza análisis factorial para identificar los rasgos de personalidad, destrezas, habilidades, actitudes y aptitudes de las personas. Para lograrlo, se evalúa un grupo de personas que se desempeña en los principales roles de un equipo de desarrollo de software (director del proyecto, coordinador del proyecto, analista, desarrollador y tester) considerados empleados exitosos. Para obtener cada competencia se utilizan indicadores conductuales para medir el nivel de presencia o ausencia en la persona evaluada, según los resultados de las pruebas psicotécnicas. Las pruebas psicotécnicas incluyen los indicadores bajos y altos para clasificar la persona con un desempeño sobresaliente (Spencer y Spencer, 1993; Furnham, 2001; Bano et al., 2016). La selección de las competencias se realiza considerando los comportamientos elementales que deben demostrar los ingenieros que ocupan los roles identificados y las competencias establecidas en el núcleo de la Esencia de Semat. A continuación, se presentan el nombre y la descripción de las competencias identificadas:

Comunicación: capacidad para comunicarse oportunamente con el cliente interno y externo, mediante habilidades de escucha, construcción de preguntas y exposición de aspectos; la persona realiza intervenciones acertadas, comprendiendo las necesidades del otro y estableciendo relaciones cordiales y recíprocas que faciliten el nivel de respuesta y la negociación.

Orientación al servicio: disposición para comprender, anticipar y satisfacer las necesidades del cliente con un servicio oportuno, que supere las expectativas de calidad. Implica responder e incorporar los requisitos del cliente interno y externo, generando valor en la búsqueda de su satisfacción y el mejoramiento continuo del servicio.

Pensamiento sistémico: capacidad para organizar e integrar el funcionamiento general de la empresa y de los procesos, interrelacionando los componentes y los resultados esperados, mediante un análisis profundo de todos los factores internos y externos, para así establecer estrategias que determinen la posición competitiva de la empresa frente al mercado y el cliente.

Gestión del conocimiento: capacidad para aplicar conocimientos, mediante estrategias y dominio tecnológico, investigando y compartiendo información útil para la creación y/o resolución de situaciones, que permitan la identificación de los objetivos del negocio, a partir del análisis de riesgos calculados y oportunidades para la gestión de los diversos proyectos.

Gestión del cambio: habilidad para iniciar e impulsar el cambio, de manera proactiva, frente a los nuevos retos y necesidades del entorno, asociadas con la ingeniería o reingeniería de los proyectos.

Trabajo colaborativo: capacidad para formar parte de un grupo y trabajar en conjunto, caracterizándose por participar activamente y movilizar aspectos positivos que favorezcan la conducción y el logro de las metas propuestas. Implica evidenciar compromiso y relacionarse de manera productiva, respetuosa y efectiva, comprendiendo la repercusión de las propias acciones sobre el éxito de la labor de los demás.

Orientación al logro: capacidad de encaminar los actos a la consecución de las metas propuestas mediante la administración de los procesos establecidos, el seguimiento y control del desarrollo y la capacidad de respuesta ante diferentes situaciones que puedan intervenir en la búsqueda de los objetivos planteados. Implica crear un ambiente de trabajo que estimule la mejora, oriente la eficacia y permanente conciencia de los tiempos de entrega.

\section{Evaluación de las personas que desempeñan los roles}

La prueba 16PF-5 es una prueba psicométrica de personalidad en el campo organizacional con la que se miden 16 escalas primarias (afabilidad, razonamiento, estabilidad, dominancia, animación, atención a las normas, atrevimiento, sensibilidad, vigilancia, abstracción, privacidad, aprensión, apertura al cambio, autosuficiencia, perfeccionismo y tensión) y cinco dimensiones globales (extraversión, ansiedad, dureza, independencia y autocontrol; Irwing et al., 2014; Gómez, Arenas y Toro, 2018). La prueba de la figura humana es una prueba proyectiva donde las personas plasman de forma indirecta la esencia de su personalidad, permitiendo hipotetizar capacidades y competencias cognitivas e intelectuales (Sánchez y Pírela, 2012; Handelzalts y Ben-Artzy-Cohen, 2014; Da Silva y Muglia, 2016; Gigi, 2016). Con estas pruebas se evalúa la personalidad y los rasgos emocionales y sociales que implican la propia percepción; además, están menos sujetas a la falsificación y/o manipulación a los grupos de respuestas. Por ello, estas pruebas se suelen utilizar en procesos de selección para predecir comportamientos de futuros profesionales o de aspirantes a ingresar a un rol con orientación especifica (Sánchez y Pírela, 2012). 
La selección del tamaño de la muestra para aplicar las pruebas psicotécnicas es pequeña, teórica y no aleatoria (Argibay, 2009) porque se aplica a personas que desempeñan los roles y las competencias identificadas. Así, la construcción de los descriptores asociados con cada competencia se realiza mediante un análisis confirmatorio, considerando una muestra de diez veces el número de variables o ítems $(\mathrm{N}=10 \mathrm{k}$ donde k es el número de ítems y variables; Nunnally, 1978; Thorndike, 1982). Por ello, en este artículo se proponen ocho competencias y se evalúan diez personas por cada competencia para un total de 80 personas.

\section{Construcción de perfiles}

Posteriormente, las competencias encontradas en el grupo de personas que conforman el equipo de desarrollo de software se caracterizan y se representan con los elementos del núcleo de la Esencia de Semat. Con base en lo anterior, se procede a clasificar los resultados obtenidos para generar los niveles de cada una de las competencias definidas. En la Figura 4 se observan los niveles de competencia representados en el núcleo de la Esencia de Semat para la competencia comunicación.
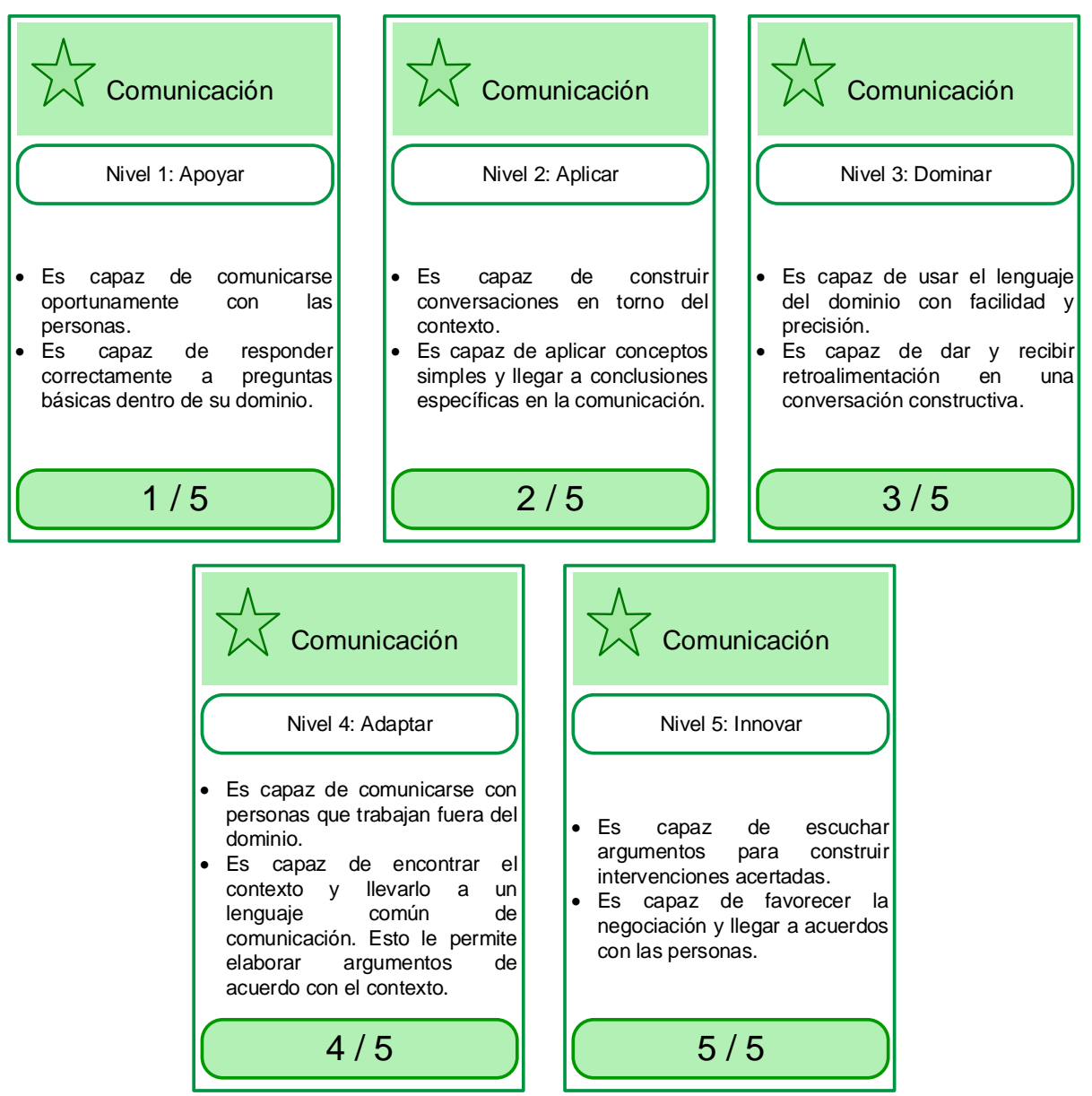

Fig. 4: Representación en el núcleo de la Esencia de Semat de los niveles de competencia para la competencia comunicación

\section{Evaluación de profesionales exitosos en los roles}

A partir de la identificación de las competencias y de los niveles de competencia se aplican las pruebas 16PF5 y figura humana a un grupo de profesionales considerados exitosos en los roles de director, coordinador, analista, desarrollador y tester. Con esta actividad se pretende la identificación de la brecha de aceptación de las competencias y de los niveles de competencia con los resultados que se obtienen en la aplicación de las pruebas psicotécnicas.

\section{Adaptación de los perfiles}

Según los resultados obtenidos en la evaluación de los profesionales exitosos en los roles, se realizan los ajusten necesarios de los perfiles según los niveles de competencia. 


\section{Definición de las competencias organizacionales de un equipo de desarrollo de software}

Se realiza la categorización de los niveles de competencia identificados para cada uno de los principales roles de un equipo de desarrollo de software: director del proyecto, coordinador del proyecto, analista del proyecto, desarrollador del proyecto y tester del proyecto (véanse las Figuras 5-7).

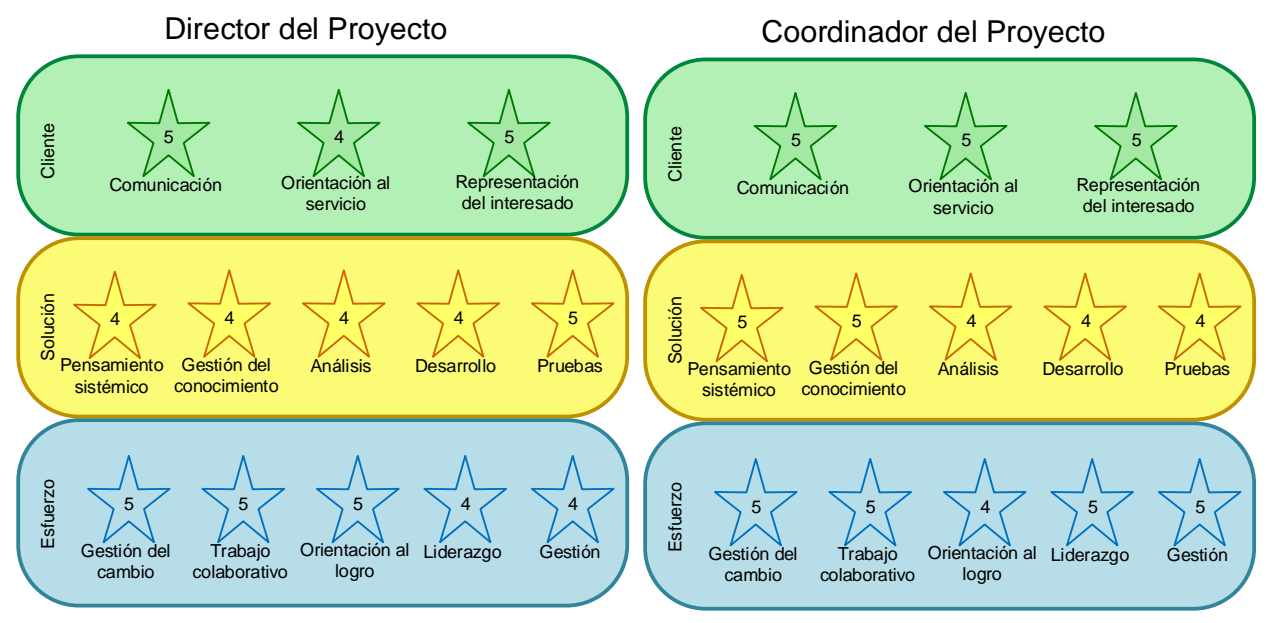

Fig. 5: Representación en el núcleo de la Esencia de Semat de las competencias y de los niveles de competencia de los roles director y coordinador del proyecto de un equipo de desarrollo de software

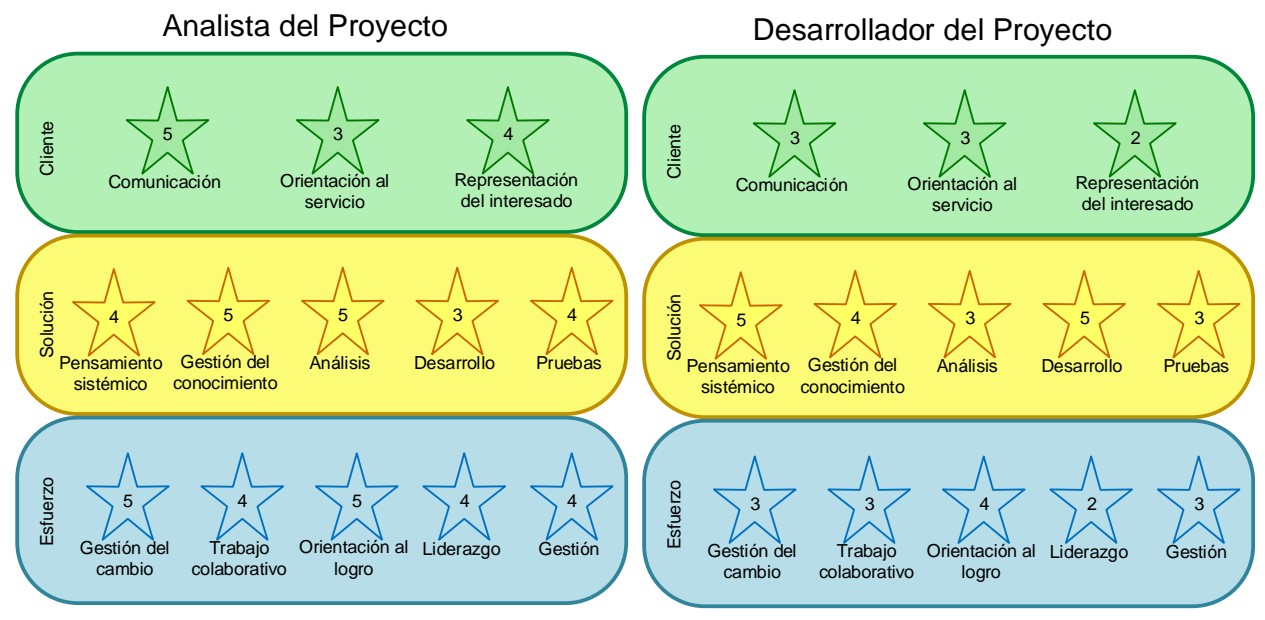

Fig. 6: Representación en el núcleo de la Esencia de Semat de las competencias y de los niveles de competencia de los roles analista y desarrollador del proyecto de un equipo de desarrollo de software

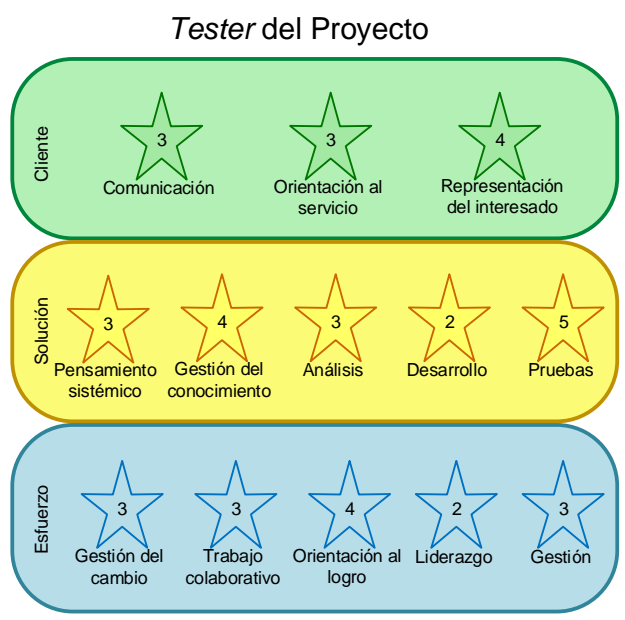

Fig. 7: Representación en el núcleo de la Esencia de Semat de las competencias y de los niveles de competencia del rol tester del proyecto de un equipo de desarrollo de software 
La percepción de aceptación de las competencias propuestas se realiza en el marco de la Tesis Doctoral "Formulación de una Teoría General para la Enseñanza de Ingeniería de Software" denominada SETMAT. La autora realiza las representaciones en SETMAT de dos cursos de ingeniería de software para universitarios, utilizando las competencias para representar buenas prácticas de enseñanza de ingeniería de software. Además, la autora realiza la validación con docentes de ingeniería de software como usuarios potenciales de SETMAT (Gómez, 2018). Como resultado se obtiene que las trece competencias son suficientes para describir las destrezas que necesitan los diferentes roles dentro de un equipo de desarrollo de software en un entorno académico. Además, dichas competencias tienen aplicabilidad en el campo empresarial. Por ello, se espera medir la capacidad productiva y el desempeño de los ingenieros que conforman equipos de desarrollo de alto rendimiento en empresas desarrolladoras de software.

\section{DISCUSIÓN Y ANÁLISIS FINAL}

En el proceso de desarrollo de software se requiere contar con equipos de trabajo con alto rendimiento y calidad. Para lograrlo, es importante identificar las personas con las habilidades requeridas para conformar equipos de trabajo según las funciones, la forma de trabajo y el tipo de proyecto de software a desarrollar. Por lo anterior, se identificaron cinco roles principales en un equipo de desarrollo de software: director del proyecto, coordinador del proyecto, analista del proyecto, desarrollador del proyecto y tester del proyecto. Posteriormente, según los principales roles se aplicaron pruebas psicotécnicas a un grupo de personas exitosas que ocupan los principales roles. Con base en los resultados de las pruebas psicotécnicas se obtuvieron siete nuevas competencias y se representaron en el núcleo de la Esencia de Semat: comunicación, orientación al servicio, pensamiento sistémico, gestión del conocimiento, gestión del cambio, trabajo colaborativo y orientación al logro. Para cada competencia se realizó la representación de sus niveles de competencia (véase la Figura 4) y para cada rol principal se realizó la representación con los niveles de competencia mínimos requeridos (véanse las Figuras 5-7).

\section{CONCLUSIONES}

De acuerdo a los resultados y análisis de la investigación se pueden extraer las siguientes dos conclusiones principales: 1) La identificación de los principales roles de un equipo de desarrollo de software ayudará a equipos de desarrollo de software a conformar equipos de trabajo con los perfiles mínimos considerando las competencias que requiere cada rol del proyecto de software identificado. 2) Con la identificación de los niveles de competencia para cada uno de los principales roles identificados se podrán conformar equipos de trabajo eficientes. Además, se podrán realizar evaluaciones continuas y planes de desarrollo para obtener rendimientos excepcionales de las personas que ocupan estos roles.

\section{AGRADECIMIENTO}

Este artículo es producto de la Tesis Doctoral Definición de buenas prácticas de desarrollo de sistemas de información geográfica utilizando el núcleo de Semat y del proyecto de investigación Competencias Semat para un equipo de desarrollo de software de la convocatoria interna del año 2016 de la Universidad de San Buenaventura Medellín con código M4820. La Universidad Nacional de Colombia, sede Medellín cofinancia el proyecto con el código Hermes 33175. También, los autores agradecen el apoyo de la Profesora María Clara Gómez Álvarez en la ejecución de pruebas psicológicas y en la validación de los perfiles y de las competencias propuestas.

\section{REFERENCIAS}

Argibay, J., Muestra en Investigación cuantitativa, Subjetividad y procesos cognitivos, 13(1), 13-29 (2009)

Bano, A., N. Salled y otros cinco autores, The effect of software engineers' personality traits on team climate and performance: A systematic literature review, doi: 10.1016/j.infsof.2016.01.006, Information and Software Technology, 73, 52-65 (2016)

Barrera, R., A. Barrera y L. Hernández, Algunas consideraciones en torno al desarrollo de habilidades del ingeniero informático y el rol de la comprensión del texto en la modelación de algoritmos computacionales, Revista Mendive Científico Pedagógica, 13(2), 112-117 (2015)

Cristancho, M., R. Bayardo y otros tres atores, Articulación de la Educación con el Mundo Productivo: Competencias Laborales Generales, 1-48, Serie Guías No 21, Ministerio de Educación Nacional, Bogotá, Colombia (2006)

Da Silva, F., C. França y otros ocho autores, Team Building Criteria in Software Projects: A Mix-Method Replicated Study, doi: 10.1016/j.infsof.2012.11.006, Information and Software Technology, 55 (7), 1316-1340 (2013)

Da Silva, K. y S. Muglia, Indicadores de Criatividade no Desenho da Figura Humana, doi: 10.1590/1982-3703001682014, Psicologia: Ciência e Profissão, 36(1), 6-19 (2016) 
Durango, C., M. Amariles y C. Zapata-Rueda, Competencias Organizacionales de un Tester de Software, para Prevenir Ataques Informáticos que utilizan Ingeniería Social, Industria 4.0 escenarios e impacto, Sello Editorial Universidad de Medellín, 1aㅡ Ed., 131-143, Medellín, Colombia (2017)

Durango, C., M. Amariles y otros cuatro autores, Representación en el Núcleo de Semat de las Competencias de Ingeniería Social Necesarias para Mejorar la Seguridad Informática, Proceedings of the Latin American Software Engineering Symposium, 41-56, Bogotá, Colombia, 19-20 noviembre (2015)

Estrada, E. y A. Peña, Influencia de los Roles de Equipo en las Actividades del Desarrollador de Software, doi: 10.26507/rei.v12n23.742, Recibe, 1(2), 17-29 (2013)

Furnham, A., Psicología Organizacional: El Comportamiento del Individuo en las Organizaciones, 1르. Ed., 1-748, Oxford University Press, México D.F., México (2001)

Gallego, M. y J. Hernández, Identificación de Factores que permitan potenciar el éxito de Proyectos de Desarrollo de Software, doi: 10.22517/23447214.9241, Scientia Et Technica, 20(1), 70-80 (2015)

Gigi, A., Human Figure Drawing (HFD) Test is affected by Cognitive Style, doi: 10.4172/2471-2701.1000111, Clinical and Experimental Psychology, 2(1), 1-4 (2016)

Gómez, A., L. Arenas y F. Toro, Rasgos de Personalidad de un Grupo de Videojugadores en la Ciudad de Manizales, doi: 10.30554/tempuspsi.1.1.2067.2018, Tempus Psicológico, 1(1), 107-125 (2018)

Gómez, M., Formulación de una Teoría General para la Enseñanza de Ingeniería de Software, Tesis de Doctorado, Universidad Nacional de Colombia, Medellín, Colombia (2018)

Handelzalts, J. e Y. Ben-Artzy-Cohen, The draw-a-person test and body images, doi: 10.1027/1192-5604/a000042, Roschachiana, 35(1), 3-22 (2014)

Irwing, P., T. Booth y M. Batey, An investigation of the Factor Structure of the 16PF, version 5: A confirmatory Factor and Invariance Analysis, doi: 10.1027/1614-0001/a000125, Journal of Individual Differences, 35(1), 38-46 (2014)

Jacobson, I., P. Ng y otros tres autores, The Essence of Software Engineering: Applying the Semat Kernel, 1를. Ed-352, Addison Wesley, New Jersey, USA (2013)

Jacobson, I., P. Ng y otros tres autores, traducido por Zapata, C. y Salazar, L., La Esencia de la Ingeniería de Software: Aplicando el Núcleo de Semat, 1를. Ed-243, Nueva Librería, Buenos Aires, Argentina (2014)

Keshav, G, S. Pawar y N. Ramrakhiyani, Role Models: Mining role transitions data in IT Project Management, IEEE International Conference on Data Science and Advanced Analytics, 508-517, Montreal, Canada, 17-19 de octubre (2016)

Licorish, S. y S. MacDonell, Communication and personality profiles of global software developers, doi: 10.1016/j.infsof.2015.02.004, Information and Software Technology, 64, 113-131 (2015)

López, Y. y M. André, Roles en el Proceso de Desarrollo de Software para las Empresas Cubanas, Ingeniería Industrial, ISSN: 0258-5960, 27(1), 31-35 (2006)

Matturro, G., F. Raschetti y C. Fontán, Soft Skills in Software Development Teams a Survey of the Points of View of Team Leaders and Team Members, $8^{\text {th }}$ International Workshop on Cooperative and Human Aspects of Software Engineering, 101-104, Florence, Italy, 6-24 de mayo (2015)

Matturro, G., Soft Skills in Software Engineering: A Study of Its Demand by Software Companies in Uruguay, 6th International Workshop on Cooperative and Human Aspects of Software Engineering, 133-136, San Francisco, USA, 25 de marzo (2013)

Mtsweni, E., T. Hörne y J. Van Der, Soft Skills for Software Project Team Members, doi: 10.7763/IJCTE.2016.V8.1035, International Journal of Computer Theory and Engineering, 8(2), 150-155 (2016)

Nunnally, J., Psychometric Theory, 2ª Ed., 1-640, McGraw-Hill, New York, USA (1978)

OMG®: Object Management Group $\AA^{\circledR}$. Essence - Kernel and Language for Software Engineering Methods Version 1.1, 44-48, Needham, USA (2015)

Rodríguez, E., Y. Pérez y otros dos autores, Perfil Ideal en Equipos de Proyectos de Desarrollo de Software, ISSN: 23062495, Serie Científica de la Universidad de las Ciencias Informáticas, 6(6), 62-74 (2013)

Sánchez, M. y L. Pírela, Estudio Psicométrico de la Prueba Figura Humana, ISSN: 1317-0570, Telos, 14(2), $210-222$ (2012)

Spencer, L. y S. Spencer, Competence at Work: Models for Superior Performance, 1ㄹ Ed., 1-372, John Wiley and Sons, New York, USA (1993)

Thorndike, R., Applied Psychometrics, 1를. Ed-390, Houghton Mifflin, Boston, USA (1982) 